\title{
A NOTE ON RUIN IN A TWO STATE MARKOV MODEL ${ }^{1}$
}

BY

\author{
ChRISTIAN WAGNER
}

\begin{abstract}
We are dealing with the ruin probability and the expected ruin time in a two state Markov model where the premium is the reciprocal of an integer and the initial surplus is a multiple of the premium.
\end{abstract}

\section{KEYWORDS}

Markov chain, Ruin time, Ruin probability.

\section{Preliminaries}

Let $t_{1}, t_{2}, \ldots, t_{n}, \ldots$ be equidistant points of time. Without loss of generality we may assume that the time interval $t_{n}-t_{n-1}=1$. We consider a risk process where, if in state one at time $t_{n}$, is payable a premium $c$, and, if in state two at time $t_{n}$, is payable a benefit of 1 . Further let $0<p_{12}<1$ be the probability to move from state 1 to state 2 in the interval $\left(t_{n}, t_{n+1}\right]$, and $0<p_{21}<1$ the probability to move from state 2 to state 1 in the interval $\left(t_{n}, t_{n+1}\right]$. The process can be described as a homogeneous MARKOV chain with two states and the transition matrix

$$
\left(\begin{array}{ll}
p_{11} & p_{12} \\
p_{21} & p_{22}
\end{array}\right)=\left(\begin{array}{cc}
1-p_{12} & p_{12} \\
p_{21} & 1-p_{21}
\end{array}\right)
$$

Define the claim size $X_{n}$ by $X_{n}=0$, if the chain is in state 1 at time $t_{n}$ and $X_{n}=1$, if the chain is in state 2 at time $t_{n}$. Starting with initial surplus $u \in \mathbb{R}$ in state $i \in\{1,2\}$, let

$$
\tau_{i}(u)=\inf \left\{n \in \mathbb{N} \mid u+n c-(1+c) \sum_{j=1}^{n} X_{j}<0\right\}
$$

\footnotetext{
1 The author is indebted to the editor and an anonymous referee for their helpful comments and some simplified derivations of formulae, especially in section 3 .
} 
be the time of ruin. We are interested in the ruin probability in infinite time $\Psi_{i}(u)=P\left(\tau_{i}(u)<\infty\right)$ and in the expected value of the ruin time, $\xi_{i}(u)=E\left(\tau_{i}(u)\right)$.

In the present paper we will assume $c=\frac{1}{N}$ with $N \in \mathbb{N}, N>1$, since in most situations we can expect $c$ to be very small compared to 1 . If there is no $\frac{1}{N}$ "close" to $c$, we have at least an upper bound for $\Psi_{i}(u)$ and a lower bound for $\xi_{i}(u)$ by choosing $\frac{1}{N}<c$. Assuming further for the rest of this paper that $u \geq 0$ is a multiple of $c$, we can write $u=\frac{k}{N}$ with $k \in \mathbb{N}_{0}$.

We will derive recursion formulae for the ruin probability and the expected value of the ruin time, and we will calculate the corresponding initial values, which are

$$
\Psi_{1}(0)=N \frac{p_{12}}{p_{21}}, \quad \xi_{1}(0)=\frac{p_{21}-p_{11}+\sigma}{p_{21}(1-\sigma)},
$$

where $\sigma$ is the only root of the polynomial $p(s)=-p_{11}+s-\left(p_{21}-p_{11}\right) s^{N}-p_{22} s^{N+1}$ in the interval $(0,1)$. Further we will give a linear approximation for the expected ruin time.

\section{A RECURSION FORMULA FOR THE RUIN PROBABILITY}

To determine the ruin probability we can adapt ideas from REINHARD and SNOUSSI (2000), who in their paper are dealing with a semi-MARKOV model. In section 4 we will give some further comments on this point.

In order to ensure $\Psi_{i}(u)<1$ we suppose a positive safety loading, i.e. we suppose $\frac{1}{N}>p_{12} / p_{21}$, the net premium for the stationary chain. We start our calculation by noting that for $u \geq 0, \Psi_{1}(u)$ and $\Psi_{2}(u)$ are connected in the following manner:

$$
\begin{aligned}
& \Psi_{1}(u)=p_{11} \Psi_{1}\left(u+\frac{1}{N}\right)+p_{12} \Psi_{2}(u-1), \\
& \Psi_{2}(u)=p_{21} \Psi_{1}\left(u+\frac{1}{N}\right)+p_{22} \Psi_{2}(u-1) .
\end{aligned}
$$

For $0 \leq u<1$ equation (4) becomes

$$
\Psi_{1}(u)-p_{11} \Psi_{1}\left(u+\frac{1}{N}\right)=p_{12} .
$$

For $1 \leq u<2$ combining (4) and (5) leads to

$$
\begin{aligned}
\Psi_{1}(u)- & p_{11} \Psi_{1}\left(u+\frac{1}{N}\right) \\
\stackrel{(6)}{=} & p_{12} p_{21} \Psi_{1}\left(u-1+\frac{1}{N}\right)+p_{12} p_{22} \\
& +p_{22}\left(\Psi_{1}(u-1)-p_{11} \Psi_{1}\left(u-1+\frac{1}{N}\right)-p_{12}\right) \\
= & p_{22} \Psi_{1}(u-1)+\left(p_{21}-p_{11}\right) \Psi_{1}\left(u-1+\frac{1}{N}\right) .
\end{aligned}
$$


Finally, for $u \geq 2$ combining (4) and (5) yields

$$
\begin{aligned}
\Psi_{1}(u)- & p_{11} \Psi_{1}\left(u+\frac{1}{N}\right) \\
= & p_{12} p_{21} \Psi_{1}\left(u-1+\frac{1}{N}\right)+p_{12} p_{22} \Psi_{2}(u-2) \\
= & p_{12} p_{21} \Psi_{1}\left(u-1+\frac{1}{N}\right) \\
& +p_{22}\left(\Psi_{1}(u-1)-p_{11} \Psi_{1}\left(u-1+\frac{1}{N}\right)\right) \\
= & p_{22} \Psi_{1}(u-1)+\left(p_{21}-p_{11}\right) \Psi_{1}\left(u-1+\frac{1}{N}\right)
\end{aligned}
$$

Writing $\Phi(k)=\Psi_{1}\left(\frac{k}{N}\right)$ we are arriving at

$$
\begin{aligned}
\Phi(k)- & p_{11} \Phi(k+1) \\
& = \begin{cases}p_{12} & \text { for } 0 \leq k<N, \\
p_{22} \Phi(k-N)+\left(p_{21}-p_{11}\right) \Phi(k-N+1) & \text { for } k \geq N .\end{cases}
\end{aligned}
$$

Given $\Phi(0)$, the $N+1$ starting values $\Phi(0), \ldots, \Phi(N)$ can be calculated immediately from (9),

$$
\Phi(k)=\frac{\Phi(0)+p_{11}^{k}-1}{p_{11}^{k}} \text { for } 0 \leq k \leq N,
$$

and in the sequel the values of $\Phi(k)$ for all $k>N$ can be calculated from the linear homogeneous difference equation of order $N+1$,

$$
\Phi(k)-p_{11} \Phi(k+1)=p_{22} \Phi(k-N)+\left(p_{21}-p_{11}\right) \Phi(k-N+1) .
$$

From the values of $\Phi(k)$ resp. $\Psi_{1}(u)$ we get the values for $\Psi_{2}(u)$ with (5).

\section{THE INITIAL VALUES FOR THE RUIN PROBABILITY}

We proceed from (9). Let $n$ be an integer greater than $N$. Then

$$
\begin{aligned}
& \Phi(n)-\Phi(0) \\
& =\sum_{k=1}^{n} \Phi(k)-\Phi(k-1) \\
& =\sum_{k=1}^{N} \Phi(k)-p_{11} \Phi(k)-p_{12}
\end{aligned}
$$




$$
\begin{aligned}
& +\sum_{k=N+1}^{n} \Phi(k)-p_{11} \Phi(k)-p_{22} \Phi(k-1-N)-\left(p_{21}-p_{11}\right) \Phi(k-N) \\
= & \left(1-p_{11}\right) \sum_{k=1}^{n} \Phi(k)-N p_{12}-\sum_{j=1}^{n-N} p_{22} \Phi(j-1)+\left(p_{21}-p_{11}\right) \Phi(j) \\
= & \left(1-p_{11}\right)\left(\sum_{k=1}^{n} \Phi(k)-\sum_{k=1}^{n-N} \Phi(k)\right)-N p_{12}+p_{22} \sum_{j=1}^{n-N} \Phi(j)-\Phi(j-1) \\
= & \left(1-p_{11}\right) \sum_{k=n-N+1}^{n} \Phi(k)-N p_{12}+p_{22}(\Phi(n-N)-\Phi(0)) .
\end{aligned}
$$

Now note that $\lim _{n \rightarrow \infty} \Phi(n)=0$ because of the positive safety loading. Hence from (12) for $n \rightarrow \infty$ we obtain

$$
\Psi_{1}(0)=\Phi(0)=N \frac{p_{12}}{p_{21}} .
$$

With (5) we find $\Psi_{2}(0)=p_{21} \Psi_{1}\left(\frac{1}{N}\right)+p_{22}$, hence with (10) we get

$$
\Psi_{2}(0)=\frac{p_{12}(N-1)+p_{22}}{p_{11}} .
$$

As an example let $p_{12}=0.01$ and $p_{21}=0.2$, so the net premium is $\frac{1}{20}$. Choosing $N=10$ we obtain $\Psi_{1}(0)=0.5$ and $\Psi_{2}(0) \approx 0.9$. $\Psi_{i}(u)$ for some further values of $u$ can be found in the following table 1 .

TABLE 1

NUMERICAL EXAMPLE FOR $\Psi_{i}(u)$

\begin{tabular}{cccccc}
\hline \hline $\boldsymbol{u}$ & $\boldsymbol{\Psi}_{\mathbf{1}}(\boldsymbol{u})$ & $\boldsymbol{\Psi}_{\mathbf{2}}(\boldsymbol{u})$ & $\boldsymbol{u}$ & $\boldsymbol{\Psi}_{\mathbf{1}}(\boldsymbol{u})$ & $\boldsymbol{\Psi}_{\mathbf{2}}(\boldsymbol{u})$ \\
\hline 0.0 & 0.50000 & 0.89899 & 1.0 & 0.44714 & 0.80771 \\
0.1 & 0.49495 & 0.89797 & 2.0 & 0.39985 & 0.72531 \\
0.2 & 0.48985 & 0.89694 & 3.0 & 0.35756 & 0.65102 \\
0.3 & 0.48469 & 0.89590 & 4.0 & 0.31974 & 0.58409 \\
0.4 & 0.47949 & 0.89485 & 5.0 & 0.28591 & 0.52385 \\
0.5 & 0.47423 & 0.89378 & 6.0 & 0.25566 & 0.46967 \\
0.6 & 0.46892 & 0.89271 & 7.0 & 0.22860 & 0.42097 \\
0.7 & 0.46356 & 0.89163 & 8.0 & 0.20441 & 0.37722 \\
0.8 & 0.45814 & 0.89053 & 9.0 & 0.18277 & 0.33794 \\
0.9 & 0.45266 & 0.88943 & 10.0 & 0.16343 & 0.30268 \\
\hline \hline
\end{tabular}

Finally, look what happens if $\frac{1}{N}$ equals the net premium in the stationary chain $p_{12} / p_{21}$. As is known, with this $N$ the ruin probability is 1 (see e.g. ASMUSSEN (2000), corollary 1.12 in chapter VI). This corresponds with (13) and (14). 


\section{A RELATED MODEL AND A RISK PROCESS}

Changing the scale for the monetary unit (divide by $\frac{1}{N}$ ) for $c, u$ and $X_{j}$ we obtain an equivalent (though less obvious) formulation of the problem where the premium $c=1$ is independent of the state and $X_{j}=0$ in state $1, X_{j}=N+1$ in state 2 , and $u \in \mathbb{N}_{0}$. Now define $\hat{X}_{j}=X_{j-1}$ for $j \geq 2$. With this new claim size distribution the result $1-\hat{X}_{j}$ in a time interval may be positive only for intervals starting in state 1 . Models with this kind of restriction are considered by REINHARD and SNOUSSI as a special case of their semi-MARKOV model. Because $\hat{X}_{1}=0$ when starting in state 1 , their $\hat{\Psi}_{1}(u)$ is our $\Psi_{1}\left(\frac{u+1}{N}\right)$, in particular $\hat{\Psi}_{1}(0)=\frac{p_{12}}{p_{11}}\left(\frac{N}{p_{11}}-1\right) \approx 0.495$ in the previous example. Further their $\hat{\Psi}_{2}(u)$ is our $\Psi_{2}\left(\frac{u-N}{N}\right)$.

With $\hat{X}_{j}$ the model is closely related to a risk process with independent increments: Let $Y_{l}^{*}$ totalize the claim surplus $\hat{X}_{j}-1$ in the intervals after a stay in state 1 up to the very next stay in state 1 . Then the distribution of $Y_{l}^{*}$ is given by

$$
P\left(Y_{l}^{*}=-1\right)=p_{11}, \quad P\left(Y_{l}^{*}=n N-1\right)=p_{12} p_{22}^{n-1} p_{21}(n \in \mathbb{N})
$$

hence $Y_{1}^{*}, Y_{2}^{*}, \ldots$ are i.i.d. with expectation $E\left(Y_{l}^{*}\right)=N p_{12} / p_{21}-1<0$. Let $X_{l}^{*}=$ $Y_{l}^{*}+1$ and consider the risk process $\left\{u+n-\sum_{l=1}^{n} X_{l}^{*}\right\}_{n \geq 1}$. The result in the last interval before ruin is negative for both the risk process (ending in state 1) and the MARKOV-model with $\hat{X}_{j}$. Therefore ruin occurs in the risk process if and only if it occurs in the MARKOV-model, i.e. both have the same ruin function.

\section{A RECURSION FORMULA FOR THE EXPECTED RUIN TIME}

Here we suppose $\frac{1}{N}<p_{12} / p_{21}$. In this case of a negative safety loading ruin is certain and the expected ruin time $\xi_{i}(u), i \in\{1,2\}$, is finite.

We note that BÄUERLE (1996) showed in the context of a MARKOV-modulated model that the asymptotic behaviour of $\xi_{i}(u)$ is linear. With quite evident modifications the proof of her theorem 3.1 also works for our model. (First use the modified model as described in the beginning lines of section 4, then re-scale to premium $c$ ). The adaption of BÄUERLE's result to our situation reads

$$
\lim _{u \rightarrow \infty} \frac{\xi_{i}(u)}{u}=\frac{1}{(1+c) \eta-c}
$$

with $\eta=\lim _{j \rightarrow \infty} E\left(X_{j}\right)=p_{12} /\left(p_{12}+p_{21}\right)$, the stationary probability for state two. The method used for the calculation of the ruin probability can be adapted. We start by noting that for $u \geq 0, \xi_{1}(u)$ and $\xi_{2}(u)$ are connected in the following manner:

$$
\xi_{1}(u)=p_{11} \xi_{1}\left(u+\frac{1}{N}\right)+p_{12} \xi_{2}(u-1)+1,
$$




$$
\xi_{2}(u)=p_{21} \xi_{1}\left(u+\frac{1}{N}\right)+p_{22} \xi_{2}(u-1)+1
$$

For $0 \leq u<1$ equation (17) becomes

$$
\xi_{1}(u)-p_{11} \xi_{1}\left(u+\frac{1}{N}\right)=1
$$

For $1 \leq u<2$ combining (17) and (18) leads to

$$
\begin{aligned}
\xi_{1}(u)- & p_{11} \xi_{1}\left(u+\frac{1}{N}\right) \\
& \stackrel{(19)}{=} p_{12}\left(p_{21} \xi_{1}\left(u-1+\frac{1}{N}\right)+1\right)+1 \\
& \quad+p_{22}\left(\xi_{1}(u-1)-p_{11} \xi_{1}\left(u-1+\frac{1}{N}\right)-1\right) \\
= & p_{22} \xi_{1}(u-1)+\left(p_{21}-p_{11}\right) \xi_{1}\left(u-1+\frac{1}{N}\right)+p_{12}+p_{21}
\end{aligned}
$$

Finally, for $u \geq 2$ combining (17) and (18) yields

$$
\begin{aligned}
\xi_{1}(u)- & p_{11} \xi_{1}\left(u+\frac{1}{N}\right) \\
= & p_{12}\left(p_{21} \xi_{1}\left(u-1+\frac{1}{N}\right)+p_{22} \xi_{2}(u-2)+1\right)+1 \\
= & p_{12} p_{21} \xi_{1}\left(u-1+\frac{1}{N}\right) \\
& +p_{22}\left(\xi_{1}(u-1)-p_{11} \xi_{1}\left(u-1+\frac{1}{N}\right)-1\right)+p_{12}+1 \\
= & p_{22} \xi_{1}(u-1)+\left(p_{21}-p_{11}\right) \xi_{1}\left(u-1+\frac{1}{N}\right)+p_{12}+p_{21} .
\end{aligned}
$$

We are now re-using the symbols we introduced earlier while calculating the ruin probability. Writing $\Phi(k)=\xi_{1}\left(\frac{k}{N}\right)$ we get

$$
\begin{aligned}
& \Phi(k)-p_{11} \Phi(k+1) \\
& = \begin{cases}1 & \text { for } 0 \leq k<N, \\
p_{22} \Phi(k-N)+\left(p_{21}-p_{11}\right) \Phi(k-N+1)+p_{12}+p_{21} & \text { for } k \geq N .\end{cases}
\end{aligned}
$$

Given $\Phi(0)$, the $N+1$ starting values $\Phi(0), \ldots, \Phi(N)$ can be calculated immediately from (22),

$$
\Phi(k)=\frac{\Phi(0)+\left(p_{11}^{k}-1\right) / p_{12}}{p_{11}^{k}} \quad \text { for } 0 \leq k \leq N
$$

and in the sequel the values of $\Phi(k)$ for all $k>N$ can be calculated from the linear difference equation of order $N+1$, 


$$
\Phi(k)-p_{11} \Phi(k+1)=p_{22} \Phi(k-N)+\left(p_{21}-p_{11}\right) \Phi(k-N+1)+p_{12}+p_{21} .
$$

From the values of $\Phi(k)$ resp. $\xi_{1}(u)$ we get the values for $\xi_{2}(u)$ with (18).

Due to the above mentioned asymptotic behaviour of $\xi_{1}(u)$ we incidentally know a general solution of $(24), \Phi(k)=k /((N+1) \eta-1)+$ const. We will make use of this in section 7 .

\section{THE INITIAL VALUES FOR THE EXPECTED RUIN TIME}

For $j \in \mathbb{N}_{0}$ define $f_{j}$ as zero except for $f_{0}=p_{11}, f_{N}=p_{21}-p_{11}$ and $f_{N+1}=p_{22}$. Then we can write (22) as

$$
\Phi(k)=\sum_{j=0}^{k+1} f_{j} \Phi(k+1-j)+1-\delta_{k, N-1} f_{N} \Phi(0)+I_{\{k \geq N\}} f_{N},
$$

where $\delta_{k, N-1}$ is the KRONECKER symbol, $I_{\{k \geq N\}}$ is the indicator function and $k \in \mathbb{N}_{0}$. We are now partially following DICKSON and WATERS's (1992) calculation of the initial value for the ruin probability when the aggregate claim amount is compound PoIsson.

For $|s|<1$ we define $J(s)=\sum_{k=0}^{\infty} s^{k} \Phi(k)$. Note that since $\Phi(k)=\xi_{1}\left(\frac{k}{N}\right)=$ $O(k)$ the sum converges absolutely for every $|s|<1$. Further let $H(s)=\sum_{k=0}^{\infty} s^{k} f_{k}$.

Then

$$
\begin{aligned}
J(s)= & \sum_{k=0}^{\infty} s^{k}\left(\sum_{j=0}^{k} f_{j} \Phi(k+1-j)+f_{k+1} \Phi(0)+1\right) \\
& -s^{N-1} f_{N} \Phi(0)+\sum_{k=N}^{\infty} s^{k} f_{N} \\
= & \sum_{k=0}^{\infty} s^{k} \sum_{j=0}^{k} f_{j} \Phi(k+1-j)+\sum_{k=0}^{\infty} s^{k} f_{k+1} \Phi(0)+\frac{1}{1-s} \\
& -s^{N-1} f_{N} \Phi(0)+\frac{s^{N}}{1-s} f_{N} \\
= & \frac{1}{s} H(s)(J(s)-\Phi(0))+\Phi(0) \frac{H(s)-f_{0}}{s} \\
& -s^{N-1} f_{N} \Phi(0)+\frac{1+s^{N} f_{N}}{1-s}
\end{aligned}
$$

and hence

$$
J(s)=\frac{-\Phi(0)\left(\frac{f_{0}}{s}+s^{N-1} f_{N}\right)+\left(1+s^{N} f_{N}\right) \frac{1}{1-s}}{1-\frac{1}{s} H(s)} .
$$


Now note that $J(s)<\infty$ for all $|s|<1$. Hence, if we are putting a zero $0<\sigma<1$ of the denominator in the above formula we find that the numerator has to be zero, too. Recall that $f_{j}$ is zero except for $f_{0}=p_{11}, f_{N}=p_{21}-p_{11}$ and $f_{N+1}=p_{22}$, hence

$$
1-\frac{1}{s} H(s)=\frac{1}{s}\left(s-p_{11}-\left(p_{21}-p_{11}\right) s^{N}-p_{22} s^{N+1}\right) .
$$

No matter if $p_{21}-p_{11}$ is positive, negative or zero, we have two changes of the sign in the sequence of the coefficients of the polynomial $p(s)=-p_{11}+s-\left(p_{21}-\right.$ $\left.p_{11}\right) s^{N}-p_{22} s^{N+1}$. By DeSCARTES' rule $p(s)$ has none or two positive roots. Since $p(1)=0, p^{\prime}(1)=p_{21}-N p_{12}<0$ and $p(0)=-p_{11}<0$ there must be a second (single) root $\sigma$ less than 1. It can be determined numerically. With this $\sigma$ we get

$$
\xi_{1}(0)=\Phi(0)=\frac{p_{21}-p_{11}+\sigma}{p_{21}(1-\sigma)} .
$$

With (18) we find $\xi_{2}(0)=p_{21} \xi_{1}\left(\frac{1}{N}\right)+1$, hence with (23) we get

$$
\xi_{2}(0)=\frac{\sigma\left(p_{12}+p_{21}\right)}{p_{11}(1-\sigma)} .
$$

As an example let $p_{12}=0.025$ and $p_{21}=0.2$, so the net premium is $\frac{1}{8}$. Choosing $N=10$ we obtain $\sigma=0.994387 \ldots, \xi_{1}(0) \approx 195.5$ and $\xi_{2}(0) \approx 40.9$. $\xi_{i}(u)$ for some further values of $u$ can be found in the following table 2 .

TABLE 2

NUMERICAL EXAMPLE FOR $\xi_{j}(u)$

\begin{tabular}{cccccccccc}
\hline \hline $\boldsymbol{u}$ & $\xi_{1}(\boldsymbol{u})$ & $\bar{\xi}_{1}(\boldsymbol{u})$ & $\xi_{2}(\boldsymbol{u})$ & $\bar{\xi}_{2}(\boldsymbol{u})$ & $\boldsymbol{u}$ & $\xi_{1}(\boldsymbol{u})$ & $\bar{\xi}_{1}(\boldsymbol{u})$ & $\xi_{2}(\boldsymbol{u})$ & $\bar{\xi}_{2}(\boldsymbol{u})$ \\
\hline 0.0 & 195.5 & 194.4 & 40.9 & 23.9 & 1.0 & 240.2 & 239.4 & 82.6 & 68.9 \\
0.1 & 199.4 & 198.9 & 41.7 & 28.4 & 2.0 & 285.1 & 284.4 & 124.9 & 113.9 \\
0.2 & 203.5 & 203.4 & 42.5 & 32.9 & 3.0 & 329.9 & 329.4 & 167.8 & 158.9 \\
0.3 & 207.7 & 207.9 & 43.4 & 37.4 & 4.0 & 374.8 & 374.4 & 211.0 & 203.9 \\
0.4 & 212.0 & 212.4 & 44.3 & 41.9 & 5.0 & 419.8 & 419.4 & 254.6 & 248.9 \\
0.5 & 216.4 & 216.9 & 45.2 & 46.4 & 6.0 & 464.7 & 464.4 & 298.5 & 293.9 \\
0.6 & 221.0 & 221.4 & 46.1 & 50.9 & 7.0 & 509.6 & 509.4 & 342.6 & 338.9 \\
0.7 & 225.6 & 225.9 & 47.1 & 55.4 & 8.0 & 554.6 & 554.4 & 386.9 & 383.9 \\
0.8 & 230.4 & 230.4 & 48.0 & 59.9 & 9.0 & 599.6 & 599.4 & 431.3 & 428.9 \\
0.9 & 235.2 & 234.9 & 49.0 & 64.4 & 10.0 & 644.5 & 644.4 & 475.8 & 473.9 \\
\hline \hline
\end{tabular}

\section{AN APPROXIMATION FOR THE EXPECTED RUIN TIME}

The numerical calculation of the expected ruin time using (24) is not stable. Due to machine rounding errors, for "large" values of $k$ (in our example for about $k>2000$, i.e. $u>200$ ) it seems appropriate to use a linear approximation. 
Our aim in this section is to find $a_{i} \in \mathbb{R}(i=1,2)$ for $\tilde{\xi}_{i}(u) \stackrel{\text { def }}{=} a_{i}+u /((1+c) \eta-c)$ so that $\lim _{u \rightarrow \infty}\left\{\xi_{i}(u)-\xi_{i}(u)\right\}=0$. We first turn to $a_{1}$ and the equivalent formulation

$$
\lim _{k \rightarrow \infty}\left\{\tilde{\Phi}_{k} \stackrel{\text { def }}{=} \Phi(k)-\left(a_{1}+\frac{k}{(N+1) \eta-1}\right)\right\}=0 .
$$

Associated with the difference equations (11) and (24) is the companion matrix

$$
A=\left(\begin{array}{cccccc}
0 & 1 & 0 & \cdots & \cdots & 0 \\
0 & 0 & 1 & 0 & \cdots & 0 \\
\vdots & \vdots & \ddots & \ddots & \ddots & \vdots \\
\vdots & \vdots & & \ddots & \ddots & 0 \\
0 & 0 & \cdots & \cdots & 0 & 1 \\
-\frac{p_{22}}{p_{11}} & \frac{p_{11}-p_{21}}{p_{11}} & 0 & \cdots & 0 & \frac{1}{p_{11}}
\end{array}\right) \in \mathbb{R}^{(N+1) \times(N+1)}
$$

and the characteristic polynomial $q(s)=-p_{22}-\left(p_{21}-p_{11}\right) s+s^{N}-p_{11} s^{N+1}$ (multiplied with $\left.(-1)^{N} p_{11}\right)$. We recall our analysis of $p(s)$ from the previous section and note that, for $s \neq 0, q(s)=0$ if and only if $p(1 / s)=0$. Hence from the calculation of (29) we get the only zero $s_{1}=1 / \sigma$ of $q(s)$ with absolute value greater than 1 . This eigenvalue of $A$ makes the system unstable. Next we have $s_{2}=1$ as a single zero, which is the only zero of $p(s)$ resp. $q(s)$ on the unit circle. This can be seen with the help of the generating function $g_{X_{l}^{*}}$ of $X_{l}^{*}$ from section 4 . We have

$$
g_{X_{l}^{*}}(s)-s=\frac{p(s)}{1-p_{22} s^{N}} \text { for }|s| \leq 1
$$

Now if $p\left(e^{i \tau}\right)=0$ with $0<\tau<2 \pi$, then $g_{X_{i}^{*}}\left(e^{i \tau}\right)=e^{i \tau}$, i.e. the FOURIER transform of $X_{l}^{*}$ takes the absolute value 1. With FELLER's lemma XV.1.4 we see that this is possible only for $\tau=2 \pi n / N(n=1, \ldots, N-1)$, but then $g_{X_{l}^{*}}\left(e^{i \tau}\right)=1 \neq e^{i \tau}$. $\tilde{\Phi}_{k}, k \geq 0$, is a solution of the homogeneous system (11), which can also be written as

$$
\begin{aligned}
\left(\tilde{\Phi}_{k-N+1}, \ldots, \tilde{\Phi}_{k}, \tilde{\Phi}_{k+1}\right)^{T} & =A\left(\tilde{\Phi}_{k-N}, \ldots, \tilde{\Phi}_{k-1}, \tilde{\Phi}_{k}\right)^{T} \\
& =A^{k-N}\left(\tilde{\Phi}_{0}, \ldots, \tilde{\Phi}_{N-1}, \tilde{\Phi}_{N}\right)^{T}
\end{aligned}
$$

Since we already know that $\tilde{\Phi}_{k}$ is asymptotic (at most) linear, i.e. $\tilde{\Phi}_{k+1} / \tilde{\Phi}_{k} \rightarrow 1$ as $k \rightarrow \infty$, we see that the starting vector $\left(\tilde{\Phi}_{0}, \ldots, \tilde{\Phi}_{N-1}, \tilde{\Phi}_{N}\right)^{T}$ doesn't have a component in the direction of an eigenvector of $s_{1}=1 / \sigma$. (After a certain number of iterations roundoff errors may violate this condition and make the numerical solution grow exponentially). As shown before, the eigenvalue $s_{2}=1$ dominates the remaining eigenvalues. Hence from the JORDAN canonical form of $A$ we conclude 


$$
A^{k}\left(\tilde{\Phi}_{0}, \ldots, \tilde{\Phi}_{N-1}, \tilde{\Phi}_{N}\right)^{T} \rightarrow B\left(\tilde{\Phi}_{0}, \ldots, \tilde{\Phi}_{N-1}, \tilde{\Phi}_{N}\right)^{T} \quad \text { for } \mathrm{k} \rightarrow \infty,
$$

where $B \in \mathbb{R}^{(N+1) \times(N+1)}$ is given by $B(1, \ldots, 1)^{T}=(1, \ldots, 1)^{T}, B=x^{T} y$ with $x^{T}$ given by $A x^{T}=x^{T}$ and $y$ given by $y A=y$. We can take $x=(1, \ldots, 1)$ and $\mathrm{y}=$ $\frac{1}{N p_{12}-p_{21}}\left(p_{22}, p_{12}, \ldots, p_{12},-p_{11}\right)$. So we finally see that

$$
\tilde{\Phi}_{k} \rightarrow \frac{1}{N p_{12}-p_{21}}\left(p_{22} \tilde{\Phi}_{0}+p_{12} \tilde{\Phi}_{1}+\cdots+p_{12} \tilde{\Phi}_{N-1}-p_{11} \tilde{\Phi}_{N}\right) \text {. }
$$

Note that with the starting values (10) we would have $\tilde{\Phi}_{k} \rightarrow 0$ as expected. There remains to adjust $a_{1}$ for the current starting values. We obtain

$$
a_{1}=-\xi_{1}(0) \frac{p_{21}}{N p_{12}-p_{21}}+\frac{(N+1) N\left(p_{11}+p_{22}\right) p_{12}}{2\left(N p_{12}-p_{21}\right)^{2}} .
$$

With the help of (17) and (18) we get

$$
a_{2}=a_{1}-\frac{(N+1)\left(p_{11}-p_{21}\right)}{N p_{12}-p_{21}} .
$$

In our example we have $a_{1} \approx 194.4, a_{2} \approx 23.9$, and the approximation is better than 0.01 for about $u>34$. See also the values given for $\tilde{\xi}_{i}(u)$ in table 2 .

\section{REFERENCES}

Asmussen, S. (2000) Ruin probabilities. Advanced Series on Statistical Science and Applied probability 2, World Scientific, Singapore.

BÄUERLE, N. (1996) Some results about the expected ruin time in Markov-modulated risk models. Insurance: Mathematics and Economics 18, 119-127.

DicksON, D.C.M. and WATERS, H.R. (1992) The probability and severity of ruin in finite and infinite time. ASTIN Bulletin 22, 177-190.

Feller, W. (1971) An Introduction to Probability Theory and its Applications II, $2^{\text {nd }}$ ed., Wiley, New York.

Reinhard, J.M. and SNOussi, M. (2000) The probability of ruin in a discrete semi-Markov risk model. Blätter der Deutschen Gesellschaft für Versicherungsmathematik XXIV, 477-490.

Rolski, T., Schmidli, H., Teugels, J. and Schmidt, V. (1999) Stochastic processes for insurance and finance. Wiley Series in Probability and Statistics, Chichester.

\section{CHRISTIAN WAGNER}

Wagner \& Kunz Aktuare AG

Steinenbachgässlein 27

CH-4051 Basel

E-mail:wagner@aktuare.ch 